\title{
RNA-seq analysis of broiler liver transcriptome reveals novel responses to high ambient temperature
}

Derrick J Coble ${ }^{1 *}$, Damarius Fleming ${ }^{1}$, Michael E Persia ${ }^{1}$, Chris M Ashwell ${ }^{2}$, Max F Rothschild ${ }^{1}$, Carl J Schmidt ${ }^{3}$ and Susan J Lamont ${ }^{*}$

\begin{abstract}
Background: In broilers, high ambient temperature can result in reduced feed consumption, digestive inefficiency, impaired metabolism, and even death. The broiler sector of the U.S. poultry industry incurs approximately $\$ 52$ million in heat-related losses annually. The objective of this study is to characterize the effects of cyclic high ambient temperature on the transcriptome of a metabolically active organ, the liver. This study provides novel insight into the effects of high ambient temperature on metabolism in broilers, because it is the first reported RNA-seq study to characterize the effect of heat on the transcriptome of a metabolic-related tissue. This information provides a platform for future investigations to further elucidate physiologic responses to high ambient temperature and seek methods to ameliorate the negative impacts of heat.
\end{abstract}

Results: Transcriptome sequencing of the livers of 8 broiler males using Illumina HiSeq 2000 technology resulted in 138 million, 100-base pair single end reads, yielding a total of 13.8 gigabases of sequence. Forty genes were differentially expressed at a significance level of $P$-value $<0.05$ and a fold-change $\geq 2$ in response to a week of cyclic high ambient temperature with 27 down-regulated and 13 up-regulated genes. Two gene networks were created from the function-based Ingenuity Pathway Analysis (IPA) of the differentially expressed genes: "Cell Signaling" and "Endocrine System Development and Function". The gene expression differences in the liver transcriptome of the heat-exposed broilers reflected physiological responses to decrease internal temperature, reduce hyperthermia-induced apoptosis, and promote tissue repair. Additionally, the differential gene expression revealed a physiological response to regulate the perturbed cellular calcium levels that can result from high ambient temperature exposure.

Conclusions: Exposure to cyclic high ambient temperature results in changes at the metabolic, physiologic, and cellular level that can be characterized through RNA-seq analysis of the liver transcriptome of broilers. The findings highlight specific physiologic mechanisms by which broilers reduce the effects of exposure to high ambient temperature. This information provides a foundation for future investigations into the gene networks involved in the broiler stress response and for development of strategies to ameliorate the negative impacts of heat on animal production and welfare.

\footnotetext{
* Correspondence: djcoble@iastate.edu; sjlamont@iastate.edu

'Department of Animal Science, lowa State University, Ames, IA 50011, USA

Full list of author information is available at the end of the article
} 


\section{Background}

Heat-related stress is a key concern for the poultry industry, especially with climate change and the expansion of poultry production into regions of the world that experience extreme temperatures [1]. Heat-related stress occurs when a negative balance exists between the net energy released by a chicken into the environment, and the amount of heat energy produced [2]. This negative balance results in reduced feed consumption, digestion inefficiency, impaired metabolism, and death in broiler chickens [3-5]. St. Pierre et al. [6] estimated the annual heat-related economic loss incurred by broiler, layer, and turkey producers in the U.S. to be approximately $\$ 125$ 165 million [6]. The annual heat-related losses incurred in the broiler sector alone were estimated at $\$ 51.8$ million [6]. In the past few decades, genetic selection for broiler performance has resulted in remarkable improvements in growth rates $[7,8]$. The deleterious effects of high ambient temperature on growth rate are greater in broilers with higher growth rates than those with lower growth rates [9]. Thus, understanding the genetic basis of the physiologic response to heat is critical for improved production efficiency and welfare of poultry.

Few experiments reported to date have investigated the effects of heat on the transcriptome of chicken tissues $[10,11]$. Li et al. [10] investigated the transcriptome of broiler breast tissue in response to cyclic high ambient temperature (6-hour daily cycles of $33^{\circ} \mathrm{C}$, day 28 to 49 post-hatch) [10]. Of the 110 genes that were differentially expressed in response to high ambient temperature, four (PM20/PM21, ASB2, USP45, and TFG) were novel heatrelated stress genes. Gene ontology analysis suggested involvement of the mitogen-associated protein kinase (MAPK), ubiquitin-proteasome, and nuclear factor kappalight-chain-enhancer of activated B cells (NFKB) pathways in the response of broilers to high ambient temperature. Exposing L2 Taiwanese roosters to high ambient temperature (4-hour heat stress at $38^{\circ} \mathrm{C}$ ) resulted in the up-regulation of 169 genes and the down-regulation of 140 genes in testis tissue [11]. These genes were primarily involved in response to stress, transport, signal transduction, and metabolism.

The objective of the current study is to characterize the effects of cyclic high ambient temperature on the transcriptome of a highly metabolically active organ in broiler chicks, the liver. This is the first reported study to use RNA-seq to characterize the transcriptome of metabolic-related tissue in broilers exposed to high ambient temperature, thus providing novel insight into the effects of heat on metabolism in broilers. This information provides a platform for future investigations into the gene networks relevant to the production of commercial broilers resilient to the negative impacts of heat.

\section{Results}

Sequencing the transcriptome, aligning and mapping reads to the genome

Approximately 138 million, 100 base pair single-end reads were generated using Illumina HiSeq 2000 technology to sequence the cDNA libraries. This yielded 13.8 gigabases of total sequence and provided on average 17,249,597 reads per sample. Using the Genomic Short-Read Nucleotide Alignment Program (GSNAP) [12], 83\% or more of the reads from each sample mapped back to the reference genome after alignment. Full length (100 bp), single-end reads were used for this analysis to preserve as much sequence information as possible.

\section{Counting mapped reads}

On average, each sample had a total of 11,695,581 uniquely mapped reads. Of these reads, 2,375,095 were classified as "no feature", meaning that the reads could not be assigned to any feature in the genome. Additionally, 332,309 reads per sample were classified as ambiguous, meaning that the reads were assigned to multiple genomic features.

\section{Testing for differential expression and pathway analysis}

Forty genes were differentially expressed at a significance level of $P$-value $<0.05$ and a fold-change $\geq 2$ in response to cyclic high ambient temperature. Of these 40 genes, 27 were down-regulated and 13 up-regulated. The foldchanges induced by high ambient temperature ranged from -12.5 to 20.0 (Table 1). Two gene networks were created from the Ingenuity Pathway Analysis (IPA) of these genes: "Cell Signaling" and "Endocrine System Development and Function".

The differentially expressed gene products in the "Cell Signaling" network included BRCA1/BRCA2-containing complex, subunit 3 (BRCC3), ELKS/RAB6-interacting/ CAST family member 2 (ERC2) isoform $\mathrm{X} 1$, fibroblast growth factor 14 (FGF14), FMOD (fibromodulin), G proteincoupled receptor 133 (GPR133), LIM and senescent cell antigen-like domains 2 (LIMS2 ) isoform X2, nidogen-1 (NID1), ORM1-like 3 (Saccharomyces cerevisiae) (ORMDL3), regulatory factor $\mathrm{x}$-box binding family transcription factor member 6 (RFX6) isoform $\mathrm{X} 2$, ring finger protein 220 (RNF220), sodium channel, voltage-gated, type III, beta subunit $(S C N 3 B)$, spondin 1, extracellular matrix protein (SPON1), splA/ryanodine receptor domain and SOCS box containing 4 (SPSB4) isoform X3, and tripartite motif containing 50 (TRIM50) (Figure 1).

The "Endocrine System Development and Function" network included aldo-keto reductase family 1 , member C3 (AKR1C3), angiopoietin-like 4 (ANGPTL4), bradykinin receptor $\mathrm{B} 1$ (BDKRB1), basonuclin $1(B N C 1)$ isoform $\mathrm{X} 4$, cholecystokinin $(C C K)$, deiodinase, iodothyronine, type II (DIO2), deiodinase, iodothyronine, type III (DIO3), keratin 14 (KRT14), myosin VIIA and Rab interacting protein 
Table 1 Significance levels of differentially expressed hepatic genes in response to high ambient temperature

\begin{tabular}{|c|c|c|c|}
\hline Gene & $P$-value & Fold change & Q-value \\
\hline RFX6 & 0.01 & -12 & 0.6 \\
\hline LOC100857 & 0.007 & -11 & 0.6 \\
\hline SPSB4 & 0.001 & -11 & 0.3 \\
\hline GPR133 & 0.0005 & -11 & 0.2 \\
\hline TRPC5 & 0.02 & -11 & 0.7 \\
\hline LOC427426 & 0.02 & -11 & 0.7 \\
\hline BNC1 & 0.004 & -11 & 0.5 \\
\hline LOC423425 & 0.02 & -10 & 0.7 \\
\hline ANGPTL4 & 0.0006 & -3 & 0.2 \\
\hline LOC100859 & 0.03 & -2 & 0.7 \\
\hline LOC420770 & 0.04 & -2 & 0.7 \\
\hline LOC100858 & 0.01 & -2 & 0.7 \\
\hline KRT14 & 0.009 & -2 & 0.6 \\
\hline FMOD & 0.001 & -2 & 0.3 \\
\hline LOC419500 & 0.004 & -2 & 0.5 \\
\hline LOC100858 & 0.01 & -2 & 0.7 \\
\hline SCN3B & 0.03 & -2 & 0.7 \\
\hline S100A4 & 0.005 & -2 & 0.5 \\
\hline LOC100858 & 0.04 & -2 & 0.7 \\
\hline TRIM50 & 0.05 & -2 & 0.7 \\
\hline BRCC3 & 0.02 & -2 & 0.7 \\
\hline LOC771141 & 0.08 & -2 & 0.7 \\
\hline PDGFD & 0.03 & -2 & 0.7 \\
\hline AKR1C3 & 0.05 & -2 & 0.7 \\
\hline SPON1 & 0.03 & -2 & 0.7 \\
\hline $\mathrm{DlO} 2$ & 0.006 & -2 & 0.5 \\
\hline NID1 & 0.01 & 2 & 0.6 \\
\hline HFM1 & 0.01 & 2 & 0.6 \\
\hline BDKRB1 & 0.04 & 2 & 0.7 \\
\hline CCK & 0.04 & 2 & 0.7 \\
\hline MYRIP & 0.007 & 2 & 0.5 \\
\hline ORMDL3 & 0.05 & 2 & 0.7 \\
\hline RNF220 & 0.03 & 2 & 0.7 \\
\hline LIMS2 & 0.02 & 2 & 0.7 \\
\hline ERC2 & 0.02 & 2 & 0.7 \\
\hline S100A1 & 0.005 & 2 & 0.5 \\
\hline FGF14 & 0.008 & 2 & 0.5 \\
\hline $\mathrm{DIO} 3$ & 0.00009 & 3 & 0.1 \\
\hline LOC395159 & 0.001 & 5 & 0.3 \\
\hline LOC100857 & 0.0008 & 20 & 0.3 \\
\hline
\end{tabular}

(MYRIP), platelet derived growth factor D (PDGFD), S100 calcium binding protein A1 (S100A1), S100 calcium binding protein A4 (S100A4), and transient receptor potential cation channel, subfamily $\mathrm{C}$, member 5 (TRPC5) (Figure 2).

To determine the biological pathways elicited in the liver by exposure of the birds to heat, the differentially expressed genes were categorized by function. Cell signaling, endocrine system development and function, molecular transport, small molecule biochemistry, and vitamin and mineral metabolism were the most significant functions elicited in response to heat (Figure 3).

\section{qPCR (quantitative polymerase chain reaction)}

Five up-regulated and 4 down-regulated genes were selected for qPCR analysis ( $P$-value $\leq 0.05$, fold-change $\geq 2$ ) (Table 2). All samples represented in the RNA-seq analysis, plus samples from 8 additional broilers exposed to the same treatments were included in the qPCR analysis, resulting in 8 samples per treatment. Data from 8 of the 9 genes were in concordance between RNA-seq and qPCR analyses. For 4 genes, the qPCR differential expression between groups was in agreement with the results of the RNA-seq, and was significant. For an additional 4 genes, the relative ranking of the treatment means was the same for both qPCR and RNA-seq, but the differences were not significant for $\mathrm{qPCR}$. There was discordance between RNA-seq and $\mathrm{qPCR}$ in the relative expression levels between the temperature treatments for only one of the nine genes (LIMS2).

\section{Discussion}

The number of differentially expressed genes detected in the current study $(n=40)$ is not high, which may be because of several factors. Applying thresholds of both significance level and a minimum fold-change may have reduced the total number of genes that were declared as differentially expressed. Using four replicates of full-sib pairs of heat-exposed and control chickens reduced random genetic variation between treatment groups, and therefore may have reduced the number of differentially expressed genes occurring at random. The low number of detected differentially expressed genes is not, however, likely to be attributable to the animal number because the sample number used in this experiment is comparable to other RNA-seq experiments. Wolf and Bryk [13] used four replicates of male and female chickens to study dosage compensation in chickens using RNA-seq analysis; Ayers et al. [14] used two male and two female chickens to incorporate RNA-seq analysis to examine the sexual dimorphic gene expression before gonadal differentiation. The method used to estimate the data variance determines the level of detection of differential expression in RNA-seq data [15]. The current study has 


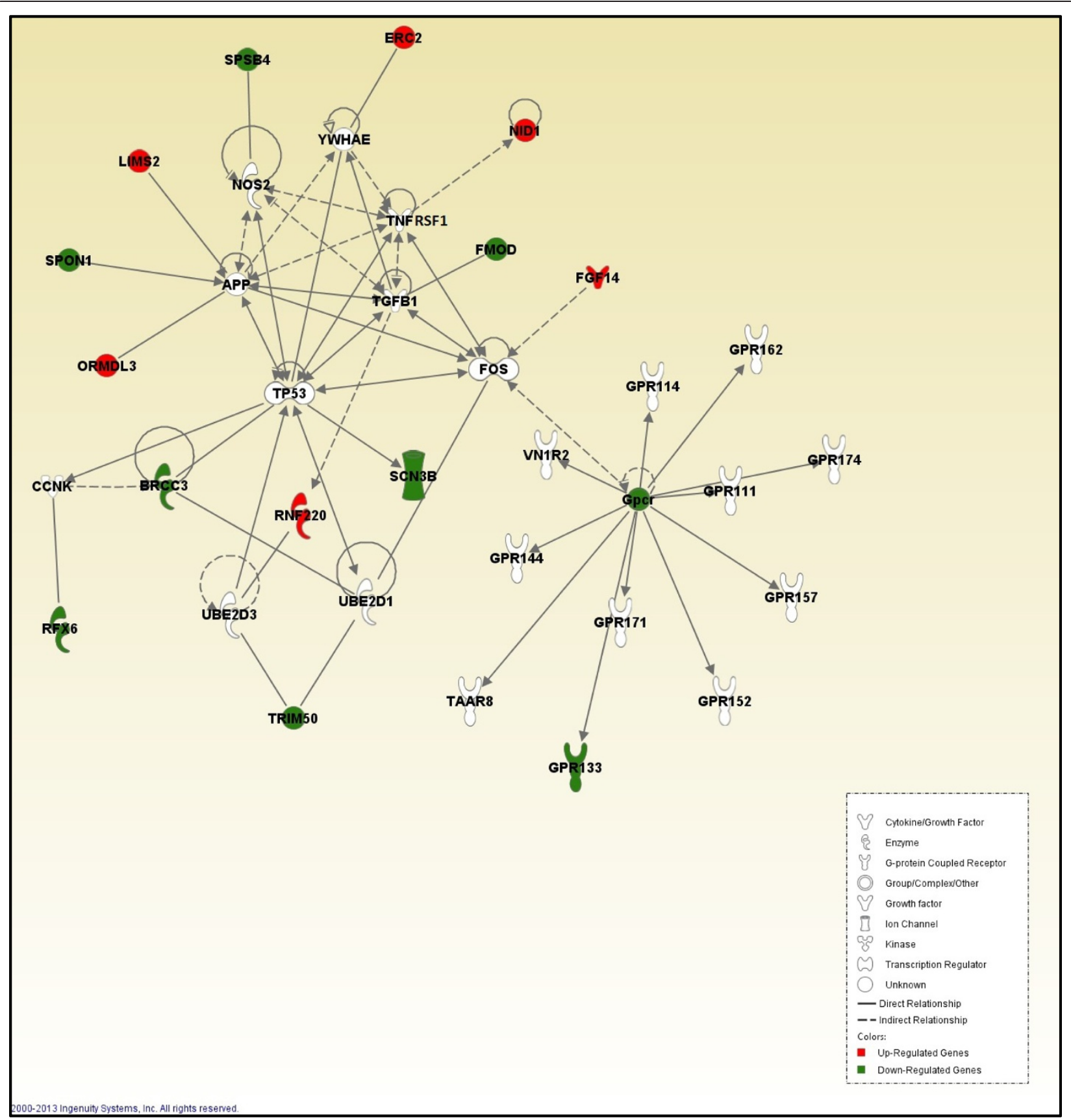

Figure 1 Cell signaling. Pathway analysis of gene functions in broiler liver transcriptome in response to cyclic high ambient temperature. Red color shows up-regulation and green color shows down-regulation (IPA). White molecules are not differentially expressed, but are included to illustrate associations with significantly up- and down-regulated genes.

increased power to determine differential expression because of the advantages of the QuasiSeq package over other methods, such as DESeq and EdgeR.

The differential expression of two genes (BRCC3 and FGF14) from the "Cell Signaling" network and five genes (CCK, TRPC5, DIO2, DIO3, and ANGPTL4) from the "Endocrine System Development and Function" network indicate that birds use specific physiologic mechanisms to regulate their internal temperature in response to high ambient temperature. CCK, DIO3, BRCC3, and FGF14 were up-regulated, while TRPC5, DIO2, and ANGPTL4 were down-regulated. CCK inhibits feed intake in chickens by promoting gastric emptying, stimulating the release of pancreatic digestive enzymes, and signaling the brainstem to depress appetite [16-18]. During exposure to heat, animals experience a negative balance in the amount of 


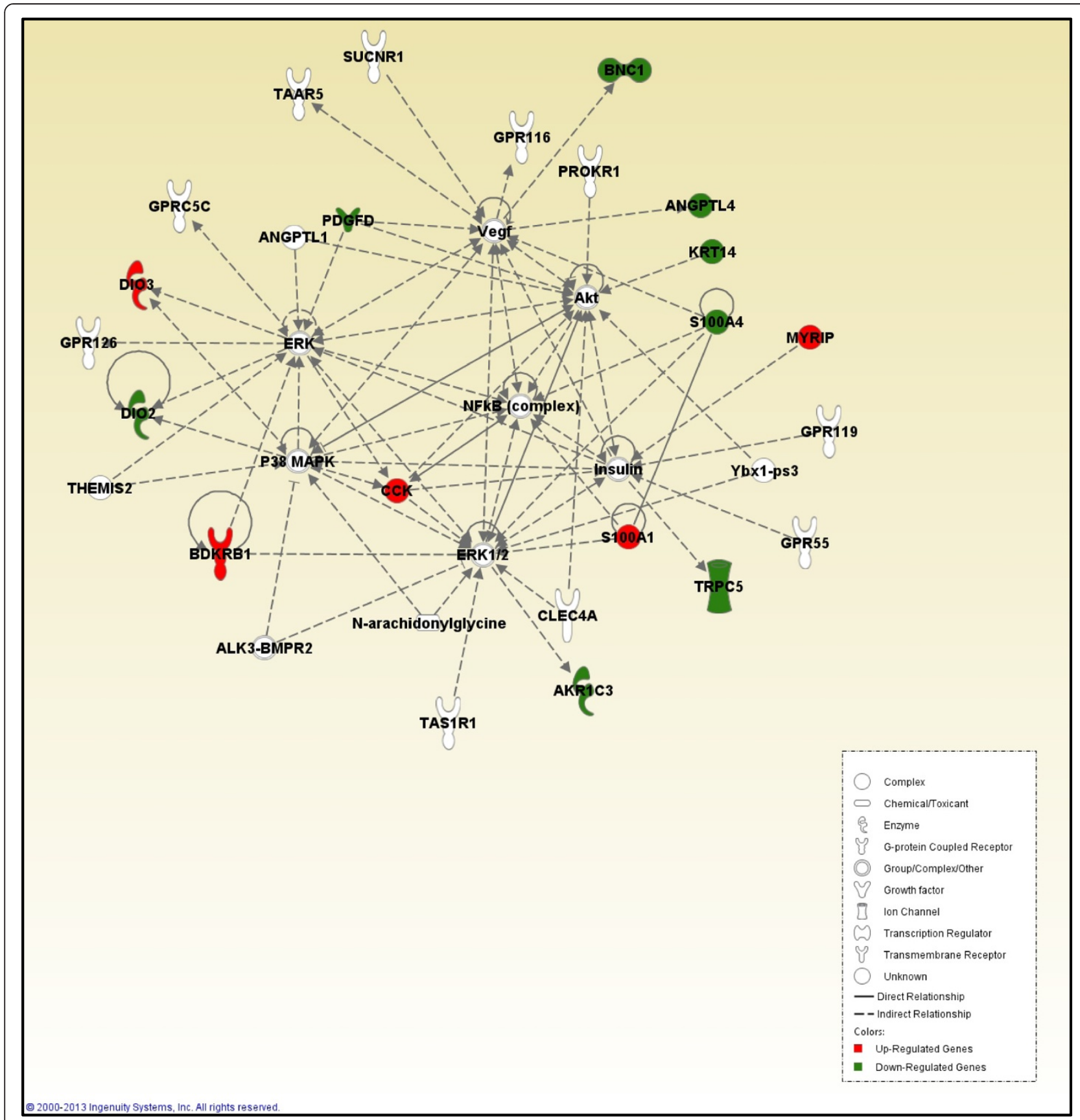

Figure 2 Endocrine system development and function. Pathway analysis of gene functions in broiler liver transcriptome in response to cyclic high ambient temperature. Red color shows up-regulation and green color shows down-regulation (IPA). White molecules are not differentially expressed, but are included to illustrate associations with significantly up- and down-regulated genes.

heat energy released and produced [2]. The inhibition of feed intake may be a mechanism to reduce the additional heat that is produced from digestive metabolism. Although the intestines are responsible for most of the body's CCK production, $C C K$ mRNA expression has also been detected in the liver [19]. Wang et al. [20] suggested that transient receptor potential channels (TRPC) are responsible for cellular CCK signaling. The differential expression of TRPC5 supports TRPC involvement in CCK signaling. $\mathrm{DIO} 2$ and $\mathrm{DIO} 3$ encode for members of an enzyme family, deiodinases, which is involved in thyroid hormone regulation [21]. Silva [22] demonstrated that thyroid hormone is involved in aerobic metabolism and thermal control. DIO2 is involved in the preservation of thyroid hormone [23], while DIO3 is associated with the inactivation of thyroid hormone [24]. Taken together, 
Figure 3 Significance levels of functions of differentially expressed hepatic genes in response to high ambient temperature. Threshold was set at $P=0.05$ and indicated as -log ( $P$-value) on the $Y$-axis. $X$-axis shows functions of differentially expressed genes.

the contrasting regulation of $\mathrm{DIO} 2$ and $\mathrm{DIO} 3$ is predicted to decrease thyroid hormone levels. Past studies have shown increases in hepatic DIO3 mRNA expression and activity levels in broilers after feed removal [25-27]. The expression patterns of CCK, TRPC5, DIO2 and DIO3 observed in the current study highlight a direct interaction between feed intake, deiodinase activity, and temperature regulation in response to the heat.

Exposure to high ambient temperature results in a redistribution of blood flow from internal organs to peripheral tissues, reducing the internal temperature of chickens [28]. $B R C C 3$, FGF14, and ANGPTL4 are all involved in regulation of blood vessel development [29-31]. Their differential expression, therefore, may be a host response to modulate internal temperature by regulating blood capillary development and distribution.

\section{Table 2 P-values from RNA-seq and qPCR validation}

\begin{tabular}{lll}
\hline Gene & RNA-seq & qPCR \\
\hline SPSB4 & 0.001 & 0.008 \\
TRPC5 & 0.02 & 0.2 \\
BNC1 & 0.004 & 0.1 \\
ANGPTL4 & 0.0006 & 0.02 \\
LIMS2 & 0.02 & 0.4 \\
ERC2 & 0.02 & 0.8 \\
S100A1 & 0.005 & 0.008 \\
FGF14 & 0.008 & 0.7 \\
DIO3 & 0.00009 & 0.02 \\
\hline
\end{tabular}

The differential expression of three genes $(S C N 3 B$, $S P O N 1$, and ORDML3) from the "Cell Signaling" network and MYRIP from the Endocrine System Development and Function" network reflects a response to hyperthermiainduced apoptosis. SCN3B, SPON1, and MYRIP were down-regulated, while $O R D M L 3$ was up-regulated in response to heat. SCN3B has been identified as a TP53inducible proapoptotic gene in mouse embryonic cells [32]. The down-regulation of $S C N 3 B$ reduces the capability of apoptosis through the TP53 pathway. SPON1 encodes for a secreted adhesion molecule that attaches to the extracellular matrix (ECM) of cells and inhibits amyloid $\beta$ (A4) precursor protein (APP) cleavage into $\beta$-secretase [33-35]. The cleavage of APP results in amyloid $\beta$ fibril formation, and induces apoptosis in yeast through mitochondrial dysfunction [36,37]. In the current study, the down-regulation of SPON1 may be a mechanism to return to homeostatic SPON1 mRNA levels following a transient up-regulation. Haughey et al. [38] suggested that amyloid $\beta$ fibril accumulation occurs within the lipid rafts, which are specialized membrane domains mainly consisting of sphingolipids and cholesterol. ORMDL3 belongs to a family of genes (ORM) that has been implicated in sphingolipid homeostasis [39]. In yeast, heat results in a transient accumulation of sphingolipid that can lead to apoptosis through sphingolipid signaling [40,41]. The upregulation of ORMDL3 reflects a cellular mechanism to regulate sphingolipid levels in response to heat, thus preventing apoptosis. MYRIP encodes for an actin motor that drives vesicle and organelle motility, and participates in endosome recycling [42]. The up-regulation of MYRIP 
may lead to enhanced ability of phagocytic cells to engulf and recycle the cellular particles after hyperthermiainduced apoptosis.

The down-regulation of three genes (FMOD, GPR133, and TRIM50) from the "Cell Signaling" network reflects the latter stages of a host-regulated tissue repair response. As a liver proteoglycan, FMOD regulates ECM organization by fibrogenic stimuli in mouse liver cells and has been described as essential for tissue repair [43]. Hepatic fibrogenesis results from damage to hepatocytes by inflammatory reactions, which alter their ECM [44]. Ruddell et al. [45] suggested that hepatic fibrogenesis was enhanced by cross-talk between hepatic lipocytes and hepatic progenitor cells (HPC), resulting in chemotaxis and cell migration. As an adhesion $G$ protein-coupled receptor (GPR), GPR133 is thought to participate in cellto-cell and cell-matrix interactions [46,47]. TRIM50 participates in inflammation suppression, fibroblast migration, and lymphocyte chemotaxis in mouse embryo fibroblasts $[48,49]$.

The up-regulation of $E R C 2$ from the "Cell Signaling" network and the differential expression of three genes (S100A1, S100A4, and BDKRB1) from the "Endocrine System Development and Function" network reflect a disruption in hepatocyte calcium levels in response to heat. $E R C 2$, S100A1, and BDKRB1 were all up-regulated, while $S 100 A 4$ was down-regulated. $E R C 2$ encodes for a protein that localizes at voltage dependent calcium channels (VDCC) [50]. The S100 family of proteins function in the regulation of calcium homeostasis, cell growth and differentiation, protein phosphorylation, and the inflammatory response [51]. The differential expression of an S100 family member (S100A11) and other genes involved in calcium-related transport and signaling was observed in catfish exposed to high ambient temperature [52]. Texel and Mattson [53] reported that excess amyloid $\beta$ fibril formation perturbed calcium homeostasis in mouse neuronal cells, possibly triggering apoptosis from calcium overload. BDKRB1 encodes for a bradykinin receptor that belongs to the rhodopsin-like GPRs that function in the regulation of inflammation [54]. The activation of $B D K R B 1$ increases cytosolic calcium levels [55]. The up-regulation of $B D K R B 1$ is a component of an inflammatory response that contributes to perturbed cellular calcium levels. The up-regulation of ERC2 and S100A1 represents calcium transport through calcium channels and calciumdependent cellular signaling. The down-regulation of S100A4 may compensate for the calcium-related transport and signaling that is carried out by the proteins encoded by $E R C 2$ and $S 100 A 1$. The differential expression of $E R C 2$, S100A1, and S100A4 illustrates the dynamic mechanisms that regulate cellular calcium levels in response to heat.

In summary, the liver transcriptome of broilers exposed to high ambient temperature indicates specific host responses to decrease internal temperature, reduce hyperthermia-induced apoptosis, and promote tissue repair. Additionally, differential expression occurred in genes that regulate the perturbed cellular calcium levels that result from exposure to heat.

\section{Conclusions}

Cyclic high ambient temperature causes metabolic, physiologic, and cellular-level changes that can be characterized through RNA-seq analysis of the liver transcriptome. The current findings suggest that these changes induce specific mechanisms by which broilers can reduce the negative physiologic effects of heat exposure. These novel insights into the effects of high ambient temperature on the metabolic transcriptome of broilers provide a foundation for future investigations into the gene networks involved in the response to heat, and for development of strategies to ameliorate the negative impacts of hot climates on animal welfare and productivity.

\section{Methods}

\section{Tissue collection}

From 22 to 28 days of age, heat-treated broilers were exposed to daily 7-hour cycles of $35^{\circ} \mathrm{C}$, while a control group was kept at $25^{\circ} \mathrm{C}$ throughout this time. The experiment was replicated with birds from different hatches. Liver samples were harvested from 4 sets of full-sibs at the midpoint of the last heat cycle on day 28 , with one bird of each pair from the high ambient temperature treatment and one from the control temperature group; these 8 samples were used for transcriptome sequencing. Liver samples were also harvested from 8 additional broilers (4 control and 4 heat-treated) from the same study, and included with the 8 samples used for RNA-seq for the qPCR validation of the transcriptome sequencing. All animal experiments were approved by the Iowa State University Institutional Animal Care and Use Committee: Log \#4-11-7128-G.

\section{Sequencing the transcriptome}

Total RNA was isolated from the liver samples following previously described procedures [56]. The RNA quality was assessed using an Agilent 2000 Bioanalyzer. Seven was the threshold RNA Integrity Number (RIN) score for cDNA library construction. The RNA was submitted to the Iowa State University DNA Facility, where libraries from each of the 8 individuals were generated and the individual liver transcriptomes were sequenced using Illumina HiSeq 2000 technology (http://www.illumina. com/systems/hiseq_2000_1000.ilmn). All 8 samples were sequenced on one lane.

\section{Quality control of RNA-seq reads}

Read quality was controlled using the FastQC suite version 0.10.1. (https://preview.iplantcollaborative.org/de/\#workspace). 
A Phred score of 28 was used to control for read quality. The adaptor sequences were removed from the reads using the fastx_clipper software of the fastx_toolkit (version 0.0.13; http://hannonlab.cshl.edu/fastx_toolkit/).

\section{Mapping reads to genome}

The RNA-seq reads were aligned using GSNAP [12], a program that can be used to align single- or paired-end reads. The authors chose to employ GSNAP over other programs because of the sequencing platform used and the advantages of using GSNAP to map RNA-Seq reads. In the current study, Illumina HiSeq 2000 technology was used to perform sequencing, resulting in 138 million, 100 bp reads. Both Illumina platforms, HiSeq 2000 and Genome Analyzer, use sequencing-by-synthesis (SBS) technology, but HiSeq 2000 has a two- to five-fold higher rate of data acquisition [57]. The ability to detect exonintron boundaries and the connections between exons is very important when assigning RNA-Seq reads to their molecules of origin. This requires the use of programs called spliced-mappers. These programs are splice-site aware and incorporate intron-like gaps. GSNAP is a splice-mapper that uses a seed-and-extend method that maps part of the gene as substrings, and then uses algorithms to extend candidate matches and locate potential splice sites [58]. This method tends to be less dependent on coverage and more likely to recover novel splice sites. The compatibility of GSNAP to the sequencing platform, the good balance between computing speed and accuracy, the availability of a reference genome, and the wide acceptance of the method are all reasons for which the authors chose GSNAP for mapping the RNA-Seq reads in the current study.

The RNA-seq reads were mapped back to the genome based on the NCBI Gallus gallus Build 4.0 reference genome. The data were run using " 32 worker threads" to optimize computational efficiency. The output was set to "split" and put into a SAM format for convenient downstream analysis. During the alignment, the "-m" setting for mismatches was set at default to allow GSNAP to auto set the number of allowed mismatches based on read length, which allowed for the best alignment across intron-exon boundaries GSNAP "soft-trims" reads during the alignment process, so that only portions of the reads above the quality threshold are aligned. Table 3 details the number of mapped and non-mapped reads, along with the number of reads before and after FastQC quality filtering (Phred 28).

\section{Counting mapped reads}

Raw reads were calculated and annotated using the HTseq package (version 4.7) in Python (http://www-huber. embl.de/users/anders/HTSeq/), which is an open source program that allows the input of raw counts from aligned reads to be annotated with gene names based on genomic features. The parameters " $\mathrm{m}<$ mode $>$ ", and "--mode = $<$ mode $>=$ " were set to "intersection non-empty". The stringency of these settings allows HT-seq to identify as many genes as possible based on overlap resolution. The parameter "-stranded" was set to "no" because the cDNA library preparation was not strand specific. The parameter "-a < minaqual" was set to "default (0)" because the read quality threshold was cutoff at Phred 28. The feature type was set at "-- type= exon" so that the reads would be counted based on exons. The GFF attribute used as the feature ID ("--idattr $=<$ id attribute $>$ ") was set to "gene_id", allowing the NCBI GFF file to output gene names. For unmapped reads, special categories were provided that explained why the reads were not mapped back to the reference genome.

\section{Testing for differential expression}

To account for the over-dispersion associated with RNA-seq data, the QuasiSeq [59] package developed in $\mathrm{R}$ [60] was used to analyze the data for differential expression. The QuasiSeq package was chosen for differential

Table 3 Number of reads before and after FastQC filtering, and number of mapped and non-mapped reads

\begin{tabular}{lllll}
\hline cDNA library & Reads (Pre-filter) & Reads (Post-filter) & Mapped reads & Non-mapped reads \\
\hline 2145 & $14,518,971$ & $14,102,129$ & $10,786,983$ & $3,315,146$ \\
2146 & $16,435,957$ & $16,079,777$ & $11,966,390$ & $4,113,387$ \\
2153 & $16,143,847$ & $15,893,619$ & $10,958,652$ & $4,934,967$ \\
2154 & $12,443,771$ & $12,157,917$ & $9,162,608$ & $2,995,309$ \\
2162 & $13,121,981$ & $12,695,265$ & $8,949,977$ & $3,745,288$ \\
2163 & $19,905,505$ & $19,516,053$ & $14,835,601$ & $4,680,452$ \\
2169 & $22,161,153$ & $21,786,920$ & $15,456,927$ & $6,329,993$ \\
2170 & $15,633,924$ & $15,633,924$ & $11,468,175$ & $4,165,749$ \\
Total & $130,365,109$ & $93,585,313$ & $34,280,291$ \\
Standard error & \pm 816.15 & $127,865,604$ & \pm 245.41 & \pm 179.13 \\
\hline
\end{tabular}


Table 4 Forward and reverse primers used for qPCR validation of RNA-seq

\begin{tabular}{lll}
\hline Gene & Forward primer & Reverse primer \\
\hline SPSB4 & 5' AATGGACTTGACCCGGAAC 3' & 5' TTCAGAGACAGAGGCAAAGG 3' \\
TRPC5 & 5' CTGCCCTGGGTTCTAGGTTT 3' & 5' GGGAGTTCATTGCAAAATCC 3' \\
BNC1 & 5' TGGATATGTGCTGCAGGATG 3' & 5' TGCCATTAACTCCACAATGG 3' \\
ANGPTL4 & 5' TGTGACATGACTGCAGAAGG 3' & 5' CAGCCAGAAGTCACCATGAA 3' \\
LIMS2 & 5' CAGATGGGCTTTTCTATGAGTT 3' & 5' GAAACATTCTGGGTGCCAGT 3' \\
ERC2 & 5' GTCTTGCCTCAACACAGCAA 3' & 5' GGCAATGTTTGCATCCTTT 3' \\
S100A1 & 5' AGCTGAGCAAGAAGGAGCTG 3' $3^{\prime}$ & 5' GGTCCTGCATGATCTTCTCC 3' \\
FGF14 & 5' TACCCAAGCCATTGGAAGTT 3' & 5' GTTTGCCGCCATTCATTATT 3' \\
DIO3 & 5' GCTCTCTTCCTTCGGGATCT 3' & 5' CCCATTCAAAATCGGTCAT 3' \\
\hline
\end{tabular}

expression analysis because of its advantages over DESeq in detecting differentially expressed genes. Upon estimating negative binomial dispersion parameters, DESeq treats the resulting estimates as constants, which can result in a non-uniform distribution of $P$-values and inaccurately estimates false discovery rates [59]. When estimating dispersions, the QuasiSeq package allows gene-specific estimates to vary around a central estimated trend and shares information across genes. When testing for differential expression, the QuasiSeq package employs a quasilikelihood method that incorporates uncertainty in the estimated variances and provides a self-tuning approach to shrinking gene-specific dispersion estimates [59]. Differential expression was declared at a significance level of $P$-value $<0.05$ and a fold-change $\geq 2$.

\section{Gene network analysis}

Upon selecting Gallus gallus in the settings, gene networks were constructed using Ingenuity Pathways Analysis [61]. Statistically significant networks were considered with a $P$-value cut-off of 0.0001 . This analysis was used to identify gene interactions within these networks.

\section{qPCR primer design}

The full cDNA sequences for each gene were obtained from NCBI. The cDNA sequences were uploaded into the NCBI Splign database and Gallus gallus was selected as the reference genome. This allowed the authors to view only the exonic regions of each gene. Sequences of 20-22 nucleotides (nt) were chosen for forward primers. These sequences were in 5'-3' (sense) orientation to the cDNA sequences and spanned exon junctions. Along with the NCBI RefSeq numbers for each gene; these forward sequences were input into Primer3, a primer design program. Primer3 is open-source software that has been widely used for primer design for over a decade [62]. It was used to select reverse sequences that were in 3'-5' (antisense) orientation to the cDNA sequences and also spanned two exons. Using primers that span exon junctions prevents the amplification of genomic DNA [63]. Primer3 allowed assessment of the quality of the primer set (melting temperature, GC\%, and self-complementarity). The output also included the product size, the sequence in FASTA format, and where the primers will anneal to the sequence. Primer3 thresholds were set to ensure a product size of $60-150 \mathrm{nt}$, a GC content of $50-60 \%$, a primer length of 18-24 nt, and a melting temperature of $60-63^{\circ} \mathrm{F}$. Primer sets that didn't span exon junctions, contained primers that were too complementary to one another, or didn't meet these specifications were rejected. Sequencing and NCBI Primer-BLAST, which checks the

Table 5 Primer set $\mathbf{r}^{2}$ values and efficiencies, along with the length of each amplicon and primer

\begin{tabular}{|c|c|c|c|c|}
\hline Primer & Length & Product size & $r^{2}$ & Efficiency \\
\hline SPSB4 For & $20 \mathrm{bp}$ & 120 bp & 1 & $100 \%$ \\
\hline SPSB4 Rev & $21 \mathrm{bp}$ & & & \\
\hline TRPC5 For & $20 \mathrm{bp}$ & 112 bp & 1 & $104 \%$ \\
\hline TRPC5 Rev & $20 \mathrm{bp}$ & & & \\
\hline BNC1 For & 20 bp & 133 bp & 1 & $109 \%$ \\
\hline BNC1 Rev & $20 \mathrm{bp}$ & & & \\
\hline ANGPTL4 For & $20 \mathrm{bp}$ & $126 \mathrm{bp}$ & 1 & $91 \%$ \\
\hline ANGPTL4 Rev & $20 \mathrm{bp}$ & & & \\
\hline LIMS2 For & $20 \mathrm{bp}$ & 143 bp & 1 & $100 \%$ \\
\hline LIMS2 Rev & $20 \mathrm{bp}$ & & & \\
\hline ERC2 For & $20 \mathrm{bp}$ & $149 \mathrm{bp}$ & 1 & $104 \%$ \\
\hline ERC2 Rev & $20 \mathrm{bp}$ & & & \\
\hline S100A1 For & $20 \mathrm{bp}$ & 120 bp & 1 & $95 \%$ \\
\hline S100A1 Rev & $20 \mathrm{bp}$ & & & \\
\hline FGF14 For & $20 \mathrm{bp}$ & 129 bp & 1 & $109 \%$ \\
\hline FGF14 Rev & $20 \mathrm{bp}$ & & & \\
\hline DIO3 For & $20 \mathrm{bp}$ & 143 bp & 1 & $104 \%$ \\
\hline DIO3 Rev & $20 \mathrm{bp}$ & & & \\
\hline
\end{tabular}


specificity of a primer set with all possible regions of the genome, were used for primer target detection.

\section{qPCR and statistical analysis}

Total RNA was isolated and quantitative PCR was performed according to previously described procedures [56]. All reactions were run in triplicate. The forward and reverse primers used for qPCR are listed in Table 4. The amplicons were verified for specificity and reaction quality using the melting curves of the reactions. The cycle threshold $(\mathrm{Ct})$ line was adjusted to fit the standard curve with an acceptable $r^{2}$ value, 0.96-1. The efficiency of each primer set was calculated using the following formula: $10^{\wedge}[(-1 /$ slope $)-1] \times 100$. Table 5 shows the $\mathrm{r}^{2}$ values, efficiencies, and NCBI RefSeq number for each primer set, along with the product length and the length of each primer. Adjusted Ct values for statistical analysis were calculated as follows: $40-[($ sample mean $\mathrm{Ct})+$ (median 28S Ct - mean $28 \mathrm{~S} \mathrm{Ct}) \times($ sample gene slope/ $28 \mathrm{~S}$ slope)] [64]. The mRNA expression levels as mean adjusted $\mathrm{Ct}$ values of each triplicate sample were analyzed using the ANOVA analysis of JMP 8.0.2 software on combined data (control and temperature-exposed broilers) for each gene separately, using the following model:

$$
\mathrm{Y}=\mu+\text { challenge }+ \text { replicate }+\mathrm{e} .
$$

Challenge and replicate were considered fixed effects. Student's t test of JMP 8.0.2 software was used to determine significant differences $(P$-value $<0.05)$ between high ambient temperature and control treatments.

\section{Data deposition}

The data discussed in this publication have been deposited in NCBI's Gene Expression Omnibus [65] and are accessible through GEO Series accession number GSE51035 (http:// www.ncbi.nlm.nih.gov/geo/query/acc.cgi?acc=GSE51035).

\section{Competing interests}

The authors declare that they have no competing interests.

\section{Authors' contributions}

DJC participated in the animal studies, participated in sample collection, carried out RNA isolation, prepared samples for RNA-seq, carried out differential expression analysis, carried out pathway analysis, and drafted the manuscript. DF participated in the animal studies, participated in sample collection, carried out RNA-seq read alignment and mapping, carried out RNA-seq read counting, and helped draft the manuscript. MEP participated in the animal studies and sample collection. CMA participated in the sample collection. MFR participated in sample collection and manuscript preparation. CJS was involved in designing the experiment. SJL designed the experiment, participated in the animal studies and sample collection, and helped draft the manuscript. All authors read and approved the final manuscript.

\section{Acknowledgements}

Supported by USDA Agriculture and Food Research Initiative Competitive Grant number 2011-67003-30228, a USDA National Needs Fellowship awarded to D. Fleming, a George Washington Carver Fellowship awarded to D.J. Coble, and Integrative Graduate Education Research and Training Fellowships awarded to D. Fleming and D. J. Coble. The authors thank the personnel that provided excellent care of the birds and skilled assistance with collection of the data and samples.

\section{Author details}

'Department of Animal Science, lowa State University, Ames, IA 50011, USA. ${ }^{2}$ Department of Poultry Science, North Carolina State University, Raleigh, NC 27695, USA. ${ }^{3}$ Department of Animal and Food Sciences, University of Delaware, Newark, DE 19716, USA.

Received: 16 August 2013 Accepted: 2 December 2014 Published: 10 December 2014

\section{References}

1. Windhorst $\mathrm{H}-\mathrm{W}$ : Changes in poultry production and trade worldwide. Worlds Poult Sci J 2006, 62(4):585-602

2. Ajakaiye J, Perez-Bello A, Mollineda-Trujillo A: Impact of heat stress on egg quality in layer hens supplemented with I-ascorbic acid and dl-tocopherol acetate. Vet Archiv 2011, 81:119-132.

3. Emmans GC, Charles DR: Climatic Environment and Poultry Feeding in Practice. 1st edition. Essex: Ancher Press Ltd; 1989.

4. Farrell DS, Swain S: Effects of temperature treatments on the energy and nitrogen metabolism of fed chickens. Br Poult Sci 1978, 18:735-748.

5. Hai $L$, Rong $D$, Zhang Z-Y: The effect of thermal environment on the digestion of broilers. Anim Physiol 2000, 83(2):57-64.

6. St. Pierre NR, Cobanov B, Schnitkey G: Economic losses from heat stress by US livestock industries. J Dairy Sci 2003, 86:E52-E77.

7. Deeb N, Cahaner A: Genotype-by-environment interaction with broiler genotypes differing in growth rate 3 . Growth rate and water consumption of broiler progeny from weight-selected vs. non-selected parents under normal and high ambient temperatures. Poult Sci 2002, 81(3):293-301.

8. McKay JC, Barton NF, Koerhuis ANM, McAdam J: The Challenge of Genetic Change in the Broiler Chicken. In The Challenge of Genetic Change in Animal Production. Edited by Hill WG, Bishop SC, Mckay JC. Edinburgh: Occasional Publishing; 2000:1-7.

9. Cahaner A, Leenstra F: Effects of high temperature on growth and efficiency of male and female broilers from lines selected for high weight gain, favorable feed conversion, and high or low fat content. Poult Sci 1992, 71(8):1237-1250.

10. Li C, Wang X, Wang G, Li N, Wu C: Expression analysis of global gene response to chronic heat exposure in broiler chickens (Gallus gallus) reveals new reactive genes. Poult Sci 2011, 90(5):1028-1036.

11. Wang SH, Cheng CY, Tang PC, Chen CF, Chen HH, Lee YP, Huang SY: Differential gene expressions in testes of L2 strain Taiwan country chicken in response to acute heat stress. Theriogenology 2013, 79(2):374-382.

12. Wu TD, Nacu S: Fast and SNP-tolerant detection of complex variants and splicing in short reads. Bioinformatics 2010, 26(7):873-881.

13. Wolf J, Bryk J: General lack of global dosage compensation in ZZ/ZW systems? Broadening the perspective with RNA-seq. BMC Genomics 2011, 12:91

14. Ayers KL, Davidson NM, Demiyah $D$, Roeszler $K N$, Grützner $F$, Sinclair $A H_{\text {, }}$ Oshlack A, Smith CA: RNA sequencing reveals sexually dimorphic gene expression before gonadal differentiation in chicken and allows comprehensive annotation of the W-chromosome. Genome Biol 2013, 14(3):1-17.

15. Wu H, Wang C, Wu Z: A new shrinkage estimator for dispersion improves differential expression detection in RNA-seq data. Biostatistics 2013, 14(2):232-243.

16. Dunn IC, Meddle SL, Wilson PW, Wardle CA, Law AS, Bishop VR, Hindar C, Robertson GW, Burt DW, Ellison SJH, Morrice DM, Hocking PM: Decreased expression of the satiety signal receptor CCKAR is responsible for increased growth and body weight during the domestication of chickens. Am J Physiol Endocrinol Metab 2013, 304(9):E909-E921.

17. Rodriguez-Sinovas A, Fernández E, Manteca X, Fernández AG, Goñalons E: CCK is involved in both peripheral and central mechanisms controlling food intake in chickens. Am J Physiol 1997, 272(1 Pt 2):R334-R340.

18. Richards MP, Proszkowiec-Weglarz M: Mechanisms regulating feed intake, energy expenditure, and body weight in poultry. Poult Sci 2007, 86(7):1478-1490. 
19. Rinn J, Rozowsky J, Laurenzi I, Petersen P, Zou K, Zhong W, Gerstein M, Snyder M: Major molecular differences between mammalian sexes are involved in drug metabolism and renal function. Dev Cell 2004, 6:791-800.

20. Wang S, Zhang AP, Kurada L, Matsui T, Lei S: Cholecystokinin facilitates neuronal excitability in the entorhinal cortex via activation of TRPC- like channels. J Neurophysiol 2011, 106(3):1515-1524.

21. Orozco A, Valverde C, Olvera A, Garcia C: lodothyronine deiodinases: a functional and evolutionary perspective. J Endocrinol 2012, 215(2):207-219.

22. Silva JE: Thyroid hormone and the energetic cost of keeping body temperature. Biosci Rep 2005, 25:129-148.

23. Peeters R, Fekete C, Goncalves C, Legradi G, Tu HM, Harney JW, Bianco AC, Lechan RM, Larsen PR: Regional physiological adaptation of the central nervous system deiodinases to iodine deficiency. Am J Physiol Endocrinol Metab 2001, 281(1):E54-E61.

24. Friesema EC, Kuiper GG, Jansen J, Visser TJ, Kester MH: Thyroid hormone transport by the human monocarboxylate transporter 8 and its rate limiting role in intracellular metabolism. Mol Endocrinol 2006, 20(11):2761-2772.

25. Darras VM, Cokelaere M, Dewil E, Arnouts S, Decuypere E, Kühn ER: Partial food restriction increases hepatic inner ring deiodinating activity in the chicken and the rat. Gen Comp Endocrinol 1995, 100(3):334-338.

26. Van der Geyten S, Van Rompaey E, Saunders JP, Visser TJ, Kühn ER, Darras VM: Regulation of thyroid hormone metabolism during fasting and refeeding in chicken. Gen Comp Endocrinol 1999, 116(2):272-280.

27. Buyse J, Decuypere E, Darras VM, Vleurick LM, Kühn ER, Veldhuis JD: Food deprivation and feeding of broiler chickens is associated with rapid and interdependent changes in the somatotrophic and thyrotrophic axes. Br Poult Sci 2000, 41(1):107-116.

28. Wolfenson D, Frei YF, Snapir N, Berman A: Heat stress effects on capillary blood flow and its redistribution in the laying hen. J Physiol 1983, 334:395-407.

29. Miskinyte S, Butler MG, Hervé D, Sarret C, Nicolino M, Petralia JD, Bergametti F, Arnould M, Pham VN, Gore AV, Spengos K, Gazal S, Woimant F, Steinberg GK, Weinstein BM, Tournier-Lasserve E: Loss of BRCC3 deubiquitinating enzyme leads to abnormal angiogenesis and is associated with syndromic moyamoya. Am J Hum Genet 2011 88(6):718-728.

30. Fields SD, Hansen PJ, Ealy AD: Fibroblast growth factor requirements for in vitro development of bovine embryos. Theriogenology 2011, 75(8):1466-1475.

31. Kersten S: Regulation of lipid metabolism via angiopoietin-like proteins. Biochem Soc Trans 2005, 33(Pt 5):1059-1062

32. Adachi K, Toyota M, Sasaki Y, Yamashita T, Ishida S, Ohe-Toyota M, Maruyama R, Hinoda $Y$, Saito T, Imai K, Kudo R, Tokino T: Identification of SCN3B as a novel p53-inducible proapoptotic gene. Oncogene 2004, 23(47):7791-7798.

33. Kitagawa M, Ao M, Miyauchi M, Abiko Y, Takata T: F-spondin regulates the differentiation of human cementoblast-like (HCEM) cells via BMP7. Biochem Biophys Res Commun 2012, 418(2):229-233.

34. Attur MG, Palmer GD, Al-Mussawir HE, Dave M, Teixeira CC, Rifkin DB, Appleton CT, Beier F, Abramson SB: F-spondin, a neuroregulatory protein, is up-regulated in osteoarthritis and regulates cartilage metabolism via TGF-beta activation. FASEB J 2009, 23(1):79-89.

35. Ho A, Südhof TC: Binding of F-spondin to amyloid-beta precursor protein: a candidate amyloid beta precursor protein ligand that modulates amyloid-beta precursor protein cleavage. Proc Natl Acad Sci 2004, 101(8):2549-2553.

36. Sakono M, Utsumi A, Zako T, Abe T, Yohda M, Maeda M: Formation of non-toxic $A \beta$ fibrils by small heat shock protein under heat-stress conditions. Biochem Biophys Res Commun 2013, 430(4):1259-1264.

37. Cha MY, Han SH, Son SM, Hong HS, Choi YJ, Byun J, Mook-Jung I: Mitochondria-specific accumulation of amyloid $\beta$ induces mitochondrial dysfunction leading to apoptotic cell death. PLOS One 2012, 7(4):e34929.

38. Haughey NJ, Bandaru WR, Bae M, Mattson MP: Roles for dysfunctional sphingolipid metabolism in Alzheimer's disease neuropathogenesis. Biochim Biophys Acta 2010, 1801(8):878-886.

39. Breslow DK, Collins SR, Bodenmiller B, Aebersold R, Simons K, Shevchenko A, Ejsing CS, Weissman JS: Orm family proteins mediate sphingolipid homeostasis. Nature 2010, 463(7284):1048-1053.

40. Sun Y, Miao Y, Yamane Y, Zhang C, Shokat KM, Takematsu H, Kozutsumi Y, Drubin DG: Orm protein phosphoregulation mediates transient sphingolipid biosynthesis response to heat stress via the Pkh-Ypk and Cdc55-PP2A pathways. Mol Biol Cell 2012, 23(12):2388-2398.

41. Nikolova-Karakashian MN, Rozenova KA: Ceramide in stress response. Adv Exp Med Biol 2010, 688:86-108.

42. Schollenberger L, Gronemeyer T, Huber CM, Lay D, Wiese S, Meyer HE, Warscheid B, Saffrich R, Peränen J, Gorgas K: RhoA regulates peroxisome association to microtubules and the actin cytoskeleton. PLOS One 2010, 5(11):e13886.

43. Mormone E, Lu Y, Ge X, Fiel MI, Nieto N: Fibromodulin, an oxidative stress-sensitive proteoglycan, regulates the fibrogenic response to liver injury in mice. Gastroenterology 2012, 142(3):612-621.

44. Gressner AM, Bachem MG: Molecular mechanisms of liver fibrogenesis-a homage to the role of activated fat-storing cells. Digestion 1995, 56(5):335-346.

45. Ruddell RG, Knight B, Tirnitz-Parker JE, Akhurst B, Summerville L, Subramaniam VN, Olynyk JK, Ramm GA: Lymphotoxin-beta receptor signaling regulates hepatic stellate cell function and wound healing in a murine model of chronic liver injury. Hepatology 2009, 49(1):227-239.

46. Yona S, Lin HH, Siu WO, Gordon S, Stacey M: Adhesion-GPCRs: emerging roles for novel receptors. Trends Biochem Sci 2008, 33(10):491-500.

47. Kwakkenbos MJ, Kop EN, Stacey M, Matmati M, Gordon S, Lin HH, Hamann J: The EGF-TM7 family: a postgenomic view. Immunogenetics 2004, 55(10):655-666.

48. Li Y, Shin D, Kwon SH: Histone deacetylase 6 plays a role as a distinct regulator of diverse cellular processes. FEBS J 2013, 280(3):775-793.

49. Fusco C, Micale L, Egorov M, Monti M, D'Addetta EV, Augello B, Cozzolino F, Calcagnì A, Fontana A, Polishchuk RS, Didelot G, Reymond A, Pucci P, Merla G: The E3-ubiquitin ligase TRIM50 interacts with HDAC6 and p62, and promotes the sequestration and clearance of ubiquitinated proteins into the aggresome. PLoS One 2012, 7(7):e40440.

50. Billings SE, Clarke GL, Nishumune $\mathrm{H}$ : ELKS1 and $\mathrm{Ca}(2+)$ channel subunit b4 interact and colocalize at cerebellar synapses. Neuroreport 2012, 23:49-54.

51. Donato R: S100: a multigenic family of calcium-modulated proteins of the EF-hand type with intracellular and extracellular functional roles. Int J Biochem Cell Biol 2001, 33(7):637-668.

52. Liu S, Wang $X$, Sun F, Zhang J, Feng J, Liu H, Rajendran KV, Sun L, Zhang Y, Jiang Y, Peatman E, Kaltenboeck L, Kucuktas H, Liu Z: RNA-Seq reveals expression signatures of genes involved in oxygen transport, protein synthesis, folding, and degradation in response to heat stress in catfish. Physiol Genomics 2013, 45(12):462-476.

53. Texel SJ, Mattson MP: Impaired adaptive cellular responses to oxidative stress and the pathogenesis of Alzheimer's disease. Antioxid Redox Signal 2011, 14(8):1519-1534.

54. Leschner J, Ring L, Feierler J, Dinkel K, Jochum M, Faussner A: Fever-like temperature modification differentially affects in vitro signaling of bradykinin $\mathrm{B}(1)$ and $\mathrm{B}(2)$ receptors. Biol Chem 2011, 392(11):1021-1029.

55. Prado GN, Taylor L, Zhou X, Ricupero D, Mierke DF, Polgar P: Mechanisms regulating the expression, self-maintenance, and signaling-function of the bradykinin B2 and B1 receptors. J Cell Physiol 2002, 193(3):275-286.

56. Cheeseman JH, Kaiser MG, Ciraci C, Kaiser P, Lamont SJ: Breed effect on early cytokine mRNA expression in spleen and cecum of chickens with and without Salmonella enteritidis infection. Dev Comp Immunol 2007, 31(1):52-60.

57. Minoche $A E$, Dohm JC, Himmelbauer H: Evaluation of genomic high-throughput sequencing data generated on Illumina HiSeq and genome analyzer systems. Genome Biol 2011, 12(11):R112.

58. Alamancos GP, Agirre E, Eyras E: Methods to study splicing from high-throughput RNA Sequencing data. Methods Mol Biol 2014, 1126:357-397.

59. Lund SP, Nettleton D, McCarthy DJ, Smyth GK: Detecting differential expression in RNA-sequence data using quasi-likelihood with shrunken dispersion estimates. Stat Appl Genet Mol Biol 2012, 11:5

60. R Development Core Team: R: A Language and Environment for Statistical Computing. Vienna, Austria: R Foundation for Statistical Computing; 2013. http://www.R-project.org/.

61. Ingenuity Pathway Analysis. http://www.ingenuity.com/.

62. Untergasser A, Cutcutache I, Koressaar T, Ye J, Faircloth BC, Remm M, Rozen SG: Primer3 - new capabilities and interfaces. Nucleic Acids Res 2012, 40:e115.

63. Ye J, Coulouris G, Zaretskaya I, Cutcutache I, Rozen S, Madden TL: Primer-BLAST: a tool to design target-specific primers for polymerase chain reaction. BMC Bioinformatics 2012, 13:134. 
64. Ciraci C, Tuggle CK, Wannemuehler MJ, Nettleton D, Lamont SJ: Unique genome-wide transcriptome profiles of chicken macrophages exposed to Salmonella-derived endotoxin. BMC Genomics 2010, 11:545.

65. Edgar R, Domrachev M, Lash AE: Gene expression omnibus: NCBI gene expression and hybridization array data repository. Nucleic Acids Res 2002, 30(1):207-210.

doi:10.1186/1471-2164-15-1084

Cite this article as: Coble et al: RNA-seq analysis of broiler liver transcriptome reveals novel responses to high ambient temperature. BMC Genomics 2014 15:1084.

\section{Submit your next manuscript to BioMed Central and take full advantage of:}

- Convenient online submission

- Thorough peer review

- No space constraints or color figure charges

- Immediate publication on acceptance

- Inclusion in PubMed, CAS, Scopus and Google Scholar

- Research which is freely available for redistribution 